\title{
Measurement of Thermal Diffusivity of Steels at Elevated Temperature by a Laser Flash Method
}

\author{
Tsuyoshi NISHI, Hiroyuki SHIBATA, ${ }^{1)}$ Koichi TSUTSUMI, ${ }^{2)}$ Hiromichi OHTA ${ }^{3)}$ and Yoshio WASEDA ${ }^{11}$ \\ Graduate School, Department of Materials Processing, Tohoku University, Sendai 980-8577 Japan. \\ E-mail: nishi@iamp.tohoku.ac.jp. 1) Institute of Multidisciplinary Research for Advanced Materials, Tohoku University, \\ Sendai 980-8577 Japan. $\quad$ 2) NKK Corporation, Materials and Processing Research Center, Fukuyama, Hiroshima $721-$ \\ 8510 Japan. $\quad 3$ 3) Department of Materials Science, Ibaraki University, Nakanarusawa, Hitachi 316-8511 Japan.
}

(Received on December 27, 2001; accepted in final form on February 2, 2002)

\begin{abstract}
In order to obtain thermal diffusivity of steels at elevated temperature with sufficient reliability using a laser flash method, an accurate value of specimen thickness is essentially required. Accordingly, a linear thermal expansion coefficient of steels was systematically measured in the temperature range from room temperature to $1676 \mathrm{~K}$. Since the decrease in specimen thickness was detected after the measurement of thermal diffusivity under vacuum at high temperature close to solidus, such a factor was quantitatively estimated from the thickness values of quenched specimens. Combining these two results, the thermal diffusivity values were successfully determined for ultra low carbon, low carbon, medium carbon, $1.2 \%$ silicon, $9 \%$ nickel and $13 \%$ chromium steels in the temperature range from room temperature to $1676 \mathrm{~K}$ using the laser flash method.
\end{abstract}

KEY WORDS: thermal diffusivity; elevated temperature; thickness of specimen; steel; linear thermal expansion coefficient; laser flash method.

\section{Introduction}

Thermal diffusivity values of steels at elevated temperatures are important to control continuous casting and hot rolling processes. Computer simulation with a model is frequently used and such works are quite useful to improve the productivity and the energy saving by optimizing the processes. In these simulation studies, accurate values of thermal properties as a function of temperatures are strongly required to improve the reliability of the model. However, the thermal diffusivity values of steels with sufficient reliability are not available at high temperature, particularly at temperature above $1400 \mathrm{~K},{ }^{1)}$ because of experimental difficulties. On the other hand, a laser flash method is well recognized as a powerful tool for measuring thermal diffusivity of a solid specimen at elevated temperature. In this laser flash measurement, an accurate value of the specimen thickness at desired temperature is essentially required to determine thermal diffusivity. However, the linear thermal expansion values of steels are not available at high temperature. ${ }^{2)}$ In addition, the experimental uncertainty of the thermal diffusivity value of steels is also affected by the change in specimen thickness at temperature near solidus under vacuum.

In order to overcome these difficulties, the linear thermal expansion values of steels are measured in the temperature range from room temperature to solidus. In addition, the variation of the specimen thickness with sufficient reliability is estimated from the thickness values of quenched speci- mens. Then, the laser flash method is also applied to three carbon steels (ultra low carbon, low carbon, and medium carbon steels) and three special steels (1.2\% silicon, 9\% nickel and $13 \%$ chromium steels). This includes the variation of the specimen thickness during the course of measurement.

The main purpose of this work is to obtain the thermal diffusivity values of commercial steels with typical chemical composition at high temperatures close to solidus systematically and to provide the recommended thermal diffusivity values as a function of temperature.

\section{Experimental Method}

Chemical compositions and some relevant properties of six steel specimens are summarized in Table 1. The per-

Table 1. Chemical composition (unit: mass\%), liquidus (TL: $\mathrm{K}$ ) and solidus (TS: K) for ultra low carbon steel (ULCS), low carbon steel (LCS), medium carbon steel (MCS), $1.2 \%$ silicon steel $(1.2 \% \mathrm{Si}), 9 \%$ nickel steel $(9 \% \mathrm{Ni})$ and $13 \%$ chromium steel $(13 \% \mathrm{Cr})$.

\begin{tabular}{|c|l|l|l|c|c|c|}
\hline composition & ULCS & LCS & MCS & $1.2 \% \mathrm{Si}$ & $9 \% \mathrm{Ni}$ & $13 \% \mathrm{Cr}$ \\
\hline $\mathrm{C}$ & 0.00017 & 0.028 & 0.135 & 0.0012 & 0.05 & 0.2 \\
$\mathrm{Si}$ & 0.01 & 0.01 & 0.32 & 1.23 & 0.25 & 0.27 \\
$\mathrm{Mn}$ & 0.17 & 0.2 & 1.35 & 0.29 & 0.58 & 0.48 \\
$\mathrm{P}$ & 0.019 & 0.008 & 0.014 & 0.041 & 0.002 & 0.009 \\
$\mathrm{~S}$ & 0.008 & 0.006 & 0.007 & - & 0.001 & 0.001 \\
sol.Al & 0.038 & 0.029 & 0.026 & 0.3 & 0.042 & 0.026 \\
\hline temperature & ULCS & LCS & MCS & $1.2 \% \mathrm{Si}$ & $9 \% \mathrm{Ni}$ & $13 \% \mathrm{Cr}$ \\
\hline $\mathrm{TL}^{11)}$ & 1807 & 1806 & 1799 & 1790 & 1773 & 1763 \\
$\mathrm{TS}^{11)}$ & 1793 & 1781 & 1758 & 1759 & 1723 & 1703 \\
\hline
\end{tabular}




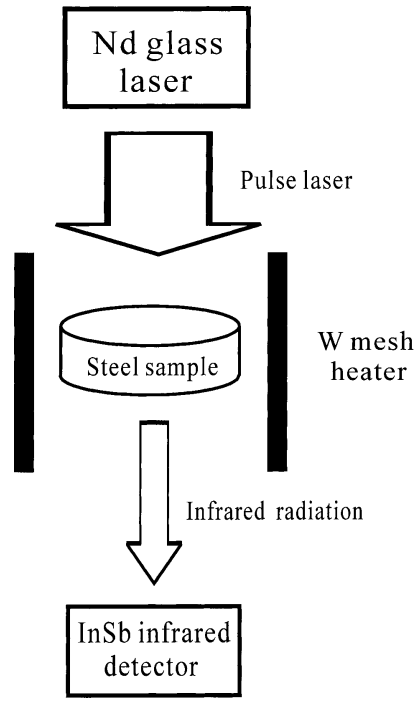

Fig. 1. Schematic diagram of a laser flash technique.

centage linear thermal expansion of the six steel specimens were measured in the temperature range from room temperature to $1676 \mathrm{~K}$ under an argon gas flow condition by using a dilatometer. The size of steel specimens for measurement of linear thermal expansion is $25 \mathrm{~mm}$ in length and $5 \mathrm{~mm}$ in diameter.

Thermal diffusivity was measured under vacuum of less than $3 \times 10^{-3} \mathrm{~Pa}$ by a laser flash method. As shown in Fig. 1, the upper surface of a specimen with a disk shape, $1 \mathrm{~mm}$ in thickness and $10 \mathrm{~mm}$ in diameter, was instantaneously irradiated by $\mathrm{Nd}$ glass laser $(10 \mathrm{~J}, 1060 \mathrm{~nm}$ emission wavelength). Then, the temperature response at the bottom surface of the specimen was measured by using an InSb infrared detector (1.2-5.5 $\mu \mathrm{m}$ effective wavelength). The specimens were heated up to $1676 \mathrm{~K}$ with a tungsten mesh heater. The specimen surface was coated with a graphite powder spray to improve the signal to noise ratio of the response curve at room temperature. On the other hand, the graphite powder spray was not utilized at elevated temperatures to keep the specimens free from contamination with carbon. In addition, the signal to noise ratio was improved in this work by accumulating ten temperature response curves.

The half-time method ${ }^{3)}$ at elevated temperature in the temperature range of above $1000 \mathrm{~K}$ is known to be, more or less, affected by radiative heat loss from the specimen surface. Then, the curve fitting method was employed in this work to determine the thermal diffusivity value of the steel specimens. The temperature rise of the rear surface, $T_{\mathrm{r}}$, is described by Eq. (1).

$$
\begin{aligned}
& T_{\mathrm{r}}=T_{\mathrm{M}} \sum_{m=0}^{\infty} A_{m} \exp \left[-\left(\frac{X_{m}}{\pi}\right)^{2} \frac{t}{t_{0}}\right] \\
& t_{0}=l^{2} /\left(\pi^{2} \alpha\right) \\
& A_{m}=2(-1)^{m} X_{m}^{2}\left(X_{m}^{2}+2 Y+Y^{2}\right)^{-1} \\
& X_{0}=(2 Y)^{1 / 2}\left(1-Y / 12+7 Y^{2} / 288\right) \\
& X_{m}=m \pi+2 Y / m \pi-4 Y^{2} /(m \pi)^{2}-2 Y^{3} / 3(m \pi)^{3} \\
& +16 Y^{4} /(m \pi)^{4} \text { for } m \geq 1
\end{aligned}
$$

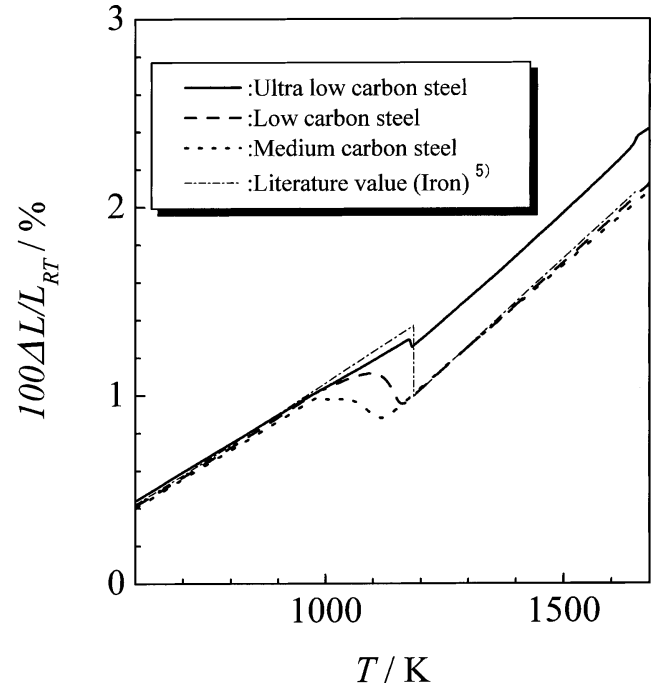

Fig. 2. Linear thermal expansion of three carbon steels as a function of temperature.

$$
Y=\frac{4 \varepsilon_{\mathrm{s}} \sigma T_{0}^{3} l}{\alpha \rho C_{p}}
$$

where $T_{\mathrm{M}}=Q / C$ is the maximum temperature rise of the rear surface of the specimen with no heat loss, $Q$ is the total energy absorbed by the specimen, $C$ is the heat capacity of the specimen, $\sigma$ is the Stefan-Boltzmann constant, $T_{0}$ is the steady-state temperature of specimen, $l$ is the thickness of specimen, $\rho$ is the density of specimen, $C_{p}$ is the specific heat capacity, and $\varepsilon_{\mathrm{s}}$ is the emissivity of the surface of specimen. Thermal diffusivity value, $\alpha$, can be obtained by fitting Eq. (1) to the measured temperature response with a least square method. The values of $Y$ and $T_{\mathrm{M}}$ are experimentally obtained from the cooling part of the measured temperature response curve. It may be worthy of note that the values of $\rho, C_{p}$ and $\varepsilon_{\mathrm{s}}$ are not required to determine the thermal diffusivity in this data processing, as already described in detail by Cezarian et al. ${ }^{4)}$

\section{Results and Discussion}

\subsection{Linear Thermal Expansion of Steels}

The linear thermal expansion values of six steels were measured in the temperature range from 603 to $1680 \mathrm{~K}$. The percentage linear thermal expansion of three carbon steels is shown in Fig. 2 as a function of temperature. The recommended values for percentage linear thermal expansion of iron $^{5)}$ were also illustrated by dashed-and-dotted line in Fig. 2. The percentage linear thermal expansion of ultra low carbon steel decreases in the temperature range from 1175 to $1180 \mathrm{~K}$. Similar behavior is detected in the temperature range from 990 to $1160 \mathrm{~K}$ for low carbon steel and that from 990 to $1115 \mathrm{~K}$ for medium carbon steel, respectively. Such decrease corresponds to the phase transformation from ferrite (bcc) phase to austenite (fcc) phase. The amount of the change of the elongation due to the transformation is less than that of the literature value of iron as shown in Fig. 2. A part of the reason of this difference may be explained by a difference of the chemical composition between the specimens used in this work and the 


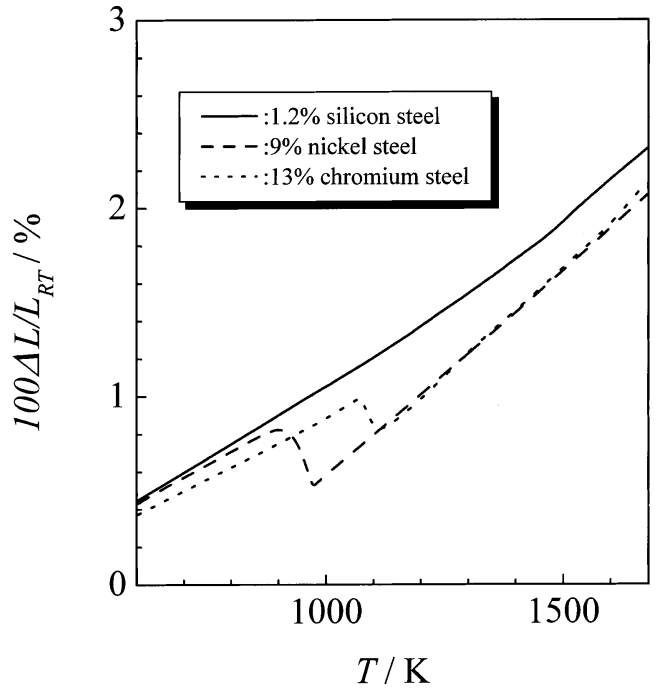

Fig. 3. Linear thermal expansion of three special steels as a function of temperature.

specimen of iron in the literature as well as the experimental conditions. However, there are no obvious explanations for the differences between them. On the other hand, the percentage linear thermal expansion of ultra low carbon steel increases in the temperature close to $1660 \mathrm{~K}$. This variation is attributed to the phase transformation from austenite (fcc) to $\delta$-ferrite (bcc) and they are coincident well with the values given in the $\mathrm{Fe}-\mathrm{C}$ phase diagram. ${ }^{\text {) }}$

The percentage linear thermal expansion of three special steels is shown in Fig. 3 as a function of temperature. The variation, corresponding to the phase transformation from ferrite (bcc) to austenite (fcc), is detected in the temperature range from 900 to $975 \mathrm{~K}$ for the $9 \%$ nickel steel and from 1070 to $1115 \mathrm{~K}$ for the $13 \%$ chromium steel, respectively. However, only the monotonic increase is observed in the percentage linear thermal expansion of the $1.2 \%$ silicon steel with increasing temperature, because only ferrite (bcc) phase can be available in the $1.2 \%$ silicon steel as confirmed by the Fe-Si phase diagram. ${ }^{7}$ The percentage linear thermal expansion, $100 \Delta L / L_{\mathrm{RT}}$, ${ }^{*}$ of six steels was fitted using a polynomial regression (fourth term), similar to the previous cases ${ }^{8,9)}$ for further analysis of thermal diffusivity as a function of $T$ in $\mathrm{K}$. The uncertainty of the linear thermal expansion of the six steels is $\pm 2 \%$, based on the previous results for silicon using the same apparatus. ${ }^{10)}$ The regression coefficients obtained are listed in Table 2.

\subsection{Estimation of the Decrease in the Specimen Thick- ness}

After the thermal diffusivity measurement close to solidus of the specimens, the reduction in both weight and thickness was clearly observed for all steel specimens. The changes are summarized in Table 3. The variation detected in thickness may be mainly due to evaporation of oxides formed on the specimen surface because the relatively low melting point is cited for these oxides. On the other hand, the change in diameter of the steel specimen was not observed before and after the measurement. In order to deter-
Table 2. Percentage linear thermal expansion values (unit: \%) of ultra low carbon steel (ULCS), low carbon steel (LCS), medium carbon steel (MCS), $1.2 \%$ silicon steel $(1.2 \% \mathrm{Si}), 9 \%$ nickel steel $(9 \% \mathrm{Ni})$ and $13 \%$ chromium steel $(13 \% \mathrm{Cr})$ fitted using a polynominal regression. The uncertainty of linear thermal expansion of six steels is suggested to be $\pm 2 \%$, based on the previous work on silicon using the same apparatus. $^{10)}$

\begin{tabular}{|c|c|c|c|c|c|c|}
\hline \multirow{2}{*}{ Steel } & Temperature & \multicolumn{5}{|c|}{ Regression $100 \Delta L / L_{R T}=\mathrm{a}_{0}+\mathrm{a}_{1} T+\mathrm{a}_{2} T^{2}+\mathrm{a}_{3} T^{3}+\mathrm{a}_{4} T^{4}$} \\
\cline { 3 - 7 } & range $(\mathrm{K})$ & $\mathrm{a}_{0}$ & $\mathrm{a}_{1}$ & $\mathrm{a}_{2}$ & $\mathrm{a}_{3}$ & $\mathrm{a}_{4}$ \\
\hline \multirow{2}{*}{$\mathrm{ULCS}$} & $603 \leqq T \leqq 1170$ & 0.347 & -0.00252 & $7.279 \times 10^{-6}$ & $-5.673 \times 10^{-9}$ & $1.605 \times 10^{-12}$ \\
\cline { 2 - 7 } & $1190 \leqq T \leqq 1640$ & -1.068 & 0.00204 & $-2.483 \times 10^{-7}$ & $1.629 \times 10^{-10}$ & $-1.875 \times 10^{-15}$ \\
\hline \multirow{2}{*}{$\mathrm{LCS}$} & $603 \leqq T \leqq 990$ & -0.215 & $7.709 \times 10^{-5}$ & $2.685 \times 10^{-6}$ & $-2.228 \times 10^{-9}$ & $7.271 \times 10^{-13}$ \\
\cline { 2 - 7 } & $1180 \leqq T \leqq 1670$ & -8.520 & 0.02160 & $-2.027 \times 10^{-5}$ & $9.324 \times 10^{-9}$ & $-1.589 \times 10^{-12}$ \\
\hline \multirow{2}{*}{$\mathrm{MCS}$} & $603 \leqq T \leqq 980$ & -0.070 & -0.00122 & $6.126 \times 10^{-6}$ & $-5.877 \times 10^{-9}$ & $2.063 \times 10^{-12}$ \\
\cline { 2 - 7 } & $1150 \leqq T \leqq 1680$ & -6.485 & 0.01601 & $-1.451 \times 10^{-5}$ & $6.709 \times 10^{-9}$ & $-1.155 \times 10^{-12}$ \\
\hline $1.2 \% \mathrm{Si}$ & $603 \leqq T \leqq 1680$ & -0.771 & 0.00267 & $-1.479 \times 10^{-6}$ & $6.820 \times 10^{-10}$ & $-5.656 \times 10^{-14}$ \\
\hline \multirow{2}{*}{$9 \% \mathrm{Ni}$} & $603 \leqq T \leqq 870$ & -9.743 & 0.05222 & $-1.033 \times 10^{-4}$ & $9.290 \times 10^{-8}$ & $3.123 \times 10^{-11}$ \\
\cline { 2 - 7 } & $990 \leqq T \leqq 1630$ & -1.531 & 0.00223 & $-2.579 \times 10^{-7}$ & $1.602 \times 10^{-10}$ & $-2.229 \times 10^{-14}$ \\
\hline \multirow{2}{*}{$13 \% \mathrm{Cr}$} & $603 \leqq T \leqq 980$ & -1.229 & 0.00518 & $-6.602 \times 10^{-6}$ & $4.748 \times 10^{-9}$ & $-1.212 \times 10^{-12}$ \\
\cline { 2 - 7 } & $1190 \leqq T \leqq 1630$ & 28.216 & -0.08823 & $1.018 \times 10^{-4}$ & $-5.059 \times 10^{-8}$ & $9.367 \times 10^{-12}$ \\
\hline
\end{tabular}

Table 3. Changes of weight and thickness for ultra low carbon steel (ULCS), low carbon steel (LCS), medium carbon steel (MCS), 1.2\% silicon steel (1.2\% Si), 9\% nicklel steel $(9 \% \mathrm{Ni})$ and $13 \%$ chromium steel $(13 \%$ $\mathrm{Cr})$.

\begin{tabular}{|c|c|c|c|c|c|c|}
\hline weight $(\mathrm{g})$ & ULCS & LCS & MCS & $1.2 \% \mathrm{Si}$ & $9 \% \mathrm{Ni}$ & $13 \% \mathrm{Cr}$ \\
\hline before meas. & 0.634 & 0.642 & 0.631 & 0.624 & 0.636 & 0.624 \\
after meas. & 0.576 & 0.589 & 0.577 & 0.568 & 0.589 & 0.562 \\
change in weight & -0.058 & -0.053 & -0.054 & -0.056 & -0.047 & -0.062 \\
\hline thickness (mm) & ULCS & LCS & MCS & $1.2 \% \mathrm{Si}$ & $9 \% \mathrm{Ni}$ & $13 \% \mathrm{Cr}$ \\
\hline before meas. & 1.030 & 1.048 & 1.035 & 1.030 & 1.032 & 1.032 \\
after meas. & 0.950 & 0.970 & 0.955 & 0.945 & 0.975 & 0.945 \\
change in thickness & -0.080 & -0.078 & -0.080 & -0.085 & -0.057 & -0.087 \\
\hline
\end{tabular}

mine thermal diffusivity, an accurate value of the specimen thickness is essentially required at desired temperature.

In order to estimate the thickness change at elevated temperature, the following procedure was employed. After heating for $30 \mathrm{~min}$ at 1572,1622 and $1676 \mathrm{~K}$, the steel specimens were quenched by furnace cooling. The quenching rate was the order of $100 \mathrm{~K} / \mathrm{s}$ at $1573 \mathrm{~K}$. The thickness of the quenched specimens was measured at room temperature using a micrometer. The true decrease in thickness of steel specimen, $\beta(\mathrm{mm})$, in the vertical axis at the elevated temperature is introduced to estimate the thickness change, and this parameter can be given by Eq. (7);

$$
\beta=l_{\mathrm{loss}}+l_{\mathrm{loss}} \times \frac{\Delta L}{L_{\mathrm{RT}}}
$$

where $l_{\text {loss }}$ is the decrease of thickness of the as-quenched specimen. The value of $\beta$ of three carbon steels is shown in Fig. 4 and that of special steels is given in Fig. 5, as a function of temperature difference defined by Ts- $T$, where Ts and $T$ are solidus ${ }^{11)}$ and measured temperature. The specimen thickness drastically decreases with increasing temperature so as to be the temperature difference, Ts- $T$, less than $200 \mathrm{~K}$. When the value of, Ts- $T$, is $100 \mathrm{~K}$, the decrease in thickness is $0.06 \mathrm{~mm}$ for the $9 \%$ nickel steel and $0.085 \mathrm{~mm}$ for other steels.

\footnotetext{
* $L_{\mathrm{RT}}$ is the length of a specimen for measuring the linear thermal expansion coefficient at room temperature, and $\Delta L$ is the elongation measured by the
} dilatometer. The expressions are not given in the two-phase region because no measurement of thermal diffusivity is made in that region. 


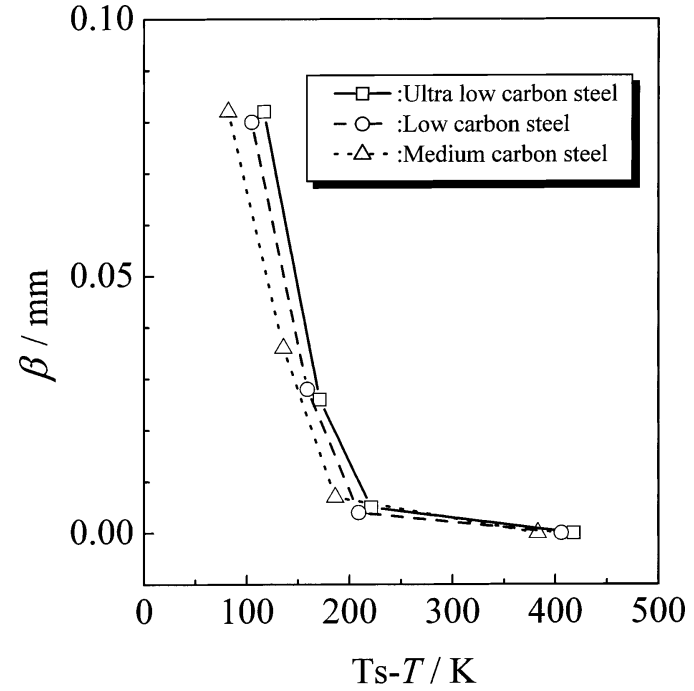

Fig. 4. Thickness change of three carbon steels as a function of temperature.

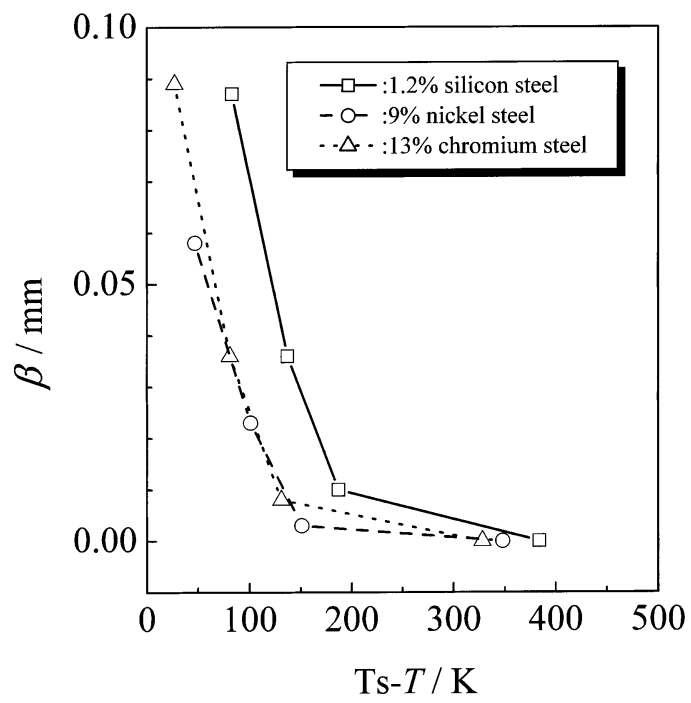

Fig. 5. Thickness change of $1.2 \%$ silicon, $9 \%$ nickel, and $13 \%$ chromium steels.

\subsection{Measurements of Thermal Diffusivity}

From the results of the linear thermal expansion coefficient and the change in thickness of steel specimens, the specimen thickness, $l_{T}$, at elevated temperature can be given by Eq. (8);

$$
l_{T}=\left(l_{\mathrm{RT}}+l_{\mathrm{RT}} \times \frac{\Delta L}{L_{\mathrm{RT}}}\right)-\beta
$$

where $l_{\mathrm{RT}}$ is the specimen thickness at room temperature. By using the relation of Eq. (19), the correction for specimen thickness is made for determing the thermal diffusivity value at temperature near solidus from measured temperature response curve. The measured temperature response curve is shown in Fig. 6 using the result of ultra low carbon steel at $773 \mathrm{~K}$ as an example. The temperature response of the bottom surface of the specimen instantaneously increases after laser irradiation, and shows the maximum value, then decreases due to the radiative heat loss from the surface of the specimen. This temperature response curve is

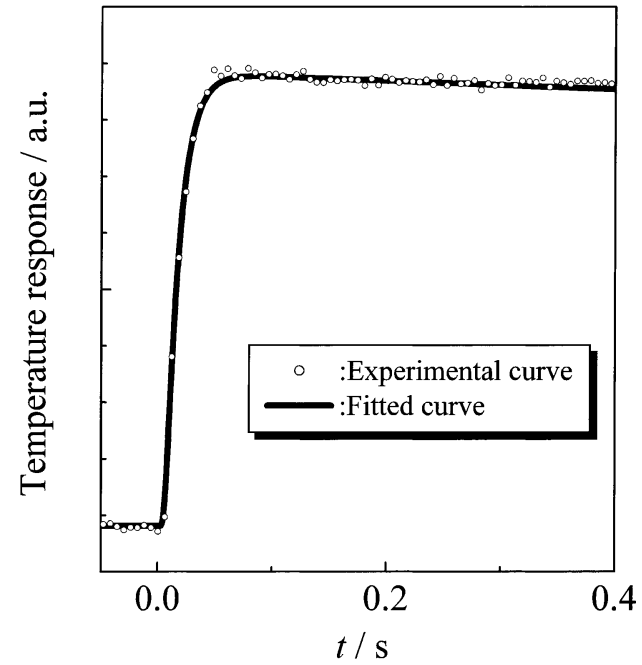

Fig. 6. Experimental and fitted temperature response curves of ultra low carbon steel at $773 \mathrm{~K}$.

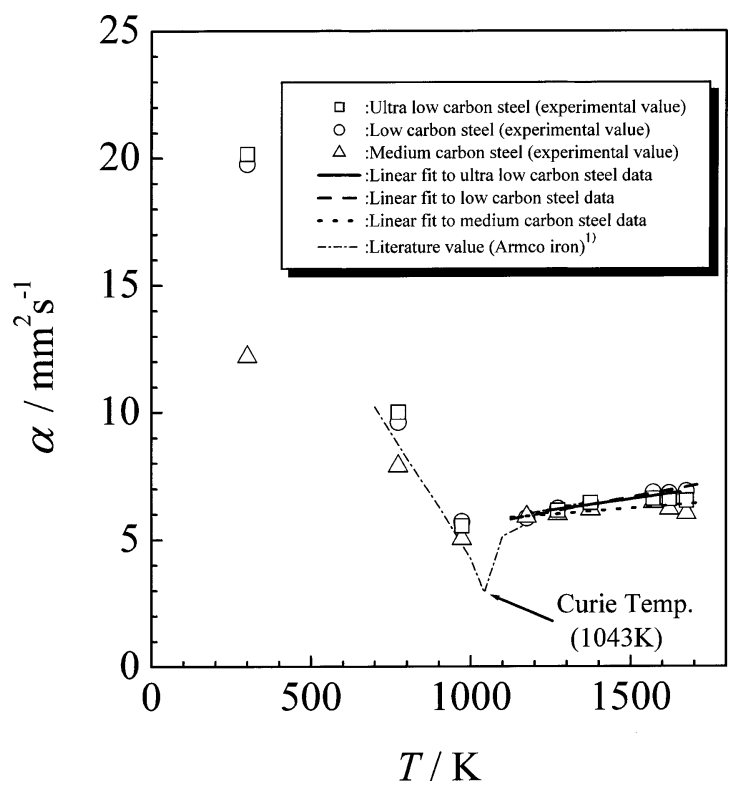

Fig. 7. Thermal diffusivity of three carbon steels as a function of temperature.

fitted by Eq. (1), then the thermal diffusivity value can be obtained. Solid line in Fig. 6 is denoted by the fitted temperature response curve which reproduces well the measured data.

The thermal diffusivity values obtained in the temperature range from room temperature to $1676 \mathrm{~K}$ are shown in Fig. 7 for ultra low carbon, low carbon, and medium carbon steels. The recommended values for thermal diffusivity of Armco iron are available in the temperature range between $700 \mathrm{~K}$ and $1400 \mathrm{~K}$. The recommended values ${ }^{1)}$ are also shown in Fig. 7. The measured values show the same tendency and good agreement with the literature values. The results are summarized as follows:

(1) The thermal diffusivity values of medium carbon steel, its carbon concentration is relatively high, is found to be smaller than those of ultra low and low carbon steels in the temperature range below ferrite-austenite $A_{1}$ transformation point $(1013 \mathrm{~K}){ }^{6}{ }^{6}$ 


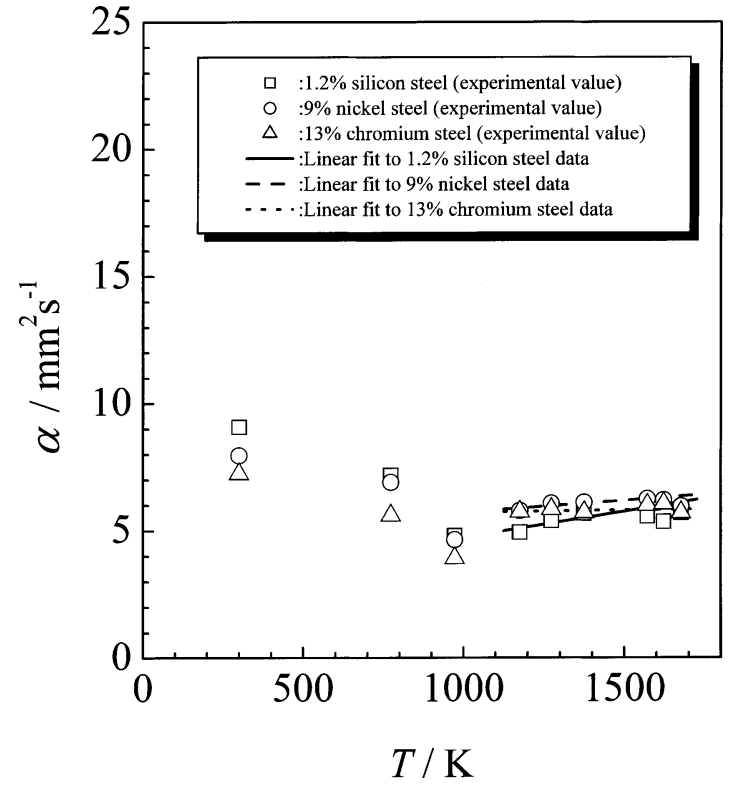

Fig. 8. Thermal diffusivity of three special steels as a function of temperature.

(2) The thermal diffusivity values of three carbon steels decreases with increasing temperature below ferriteaustenite $\mathrm{A}_{1}$ transformation point.

(3) The thermal diffusivity values of three carbon steels slightly increase with increasing temperature above ferriteaustenite A1 transformation point.

On the other hand, the thermal diffusivity values for special steels are shown in Fig. 8. The results are summarized as follows:

(1) The thermal diffusivity values of special steels decrease with increasing temperature up to $1000 \mathrm{~K}$.

(2) The thermal diffusivity values of special steels slightly increase when temperature is higher than $1000 \mathrm{~K}$. It may be noted that similar temperature dependence of thermal diffusivity are also found in several alloy specimens. ${ }^{12)}$

(3) The thermal diffusivity values of special steels are smaller than those of carbon steels in the temperature range from room temperature to $1000 \mathrm{~K}$.

\section{Summary}

In this work, the thermal diffusivity values of six steels were successfully determined by estimating the specimen thickness accurately at elevated temperature near solidus. Thermal diffusivity values of six steels are summarized in Table 4. Measured thermal diffusivity values, $\alpha$, of six steels in the temperature range of austenite phase were fitted using a linear regression. The results in the unit of $\mathrm{mm}^{2} \mathrm{~s}^{-1}$ are given below ( $T$ in $\mathrm{K}$ ) for further convenience. The regression coefficients are listed in Table $\mathbf{5}$.

The uncertainty of thermal diffusivity of six steels below $1375 \mathrm{~K}$ is $\pm 1.6 \%$. The uncertainty of thermal diffusivity of six steels above $1572 \mathrm{~K}$ increases to $\pm 5.8 \%$ because the uncertainty of $\beta$. It would be interesting to extend the present results to computer simulation for some steel making process, especially for designing the appropriate hot rolling and casting process with sufficient reliability.
Table 4. Thermal diffusivity values (unit: $\mathrm{mm}^{2} \mathrm{~s}^{-1}$ ) of ultra low carbon steel (ULCS), low carbon steel (LCS), mediumm carbon steel (MCS), $1.2 \%$ silicon steel $(1.2 \% \mathrm{Si}), 9 \%$ nickel steel $(9 \% \mathrm{Ni})$ and $13 \%$ chromium steel $(13 \% \mathrm{Cr})$.

\begin{tabular}{|c|c|c|c|c|c|c|}
\hline Temperature (K) & ULCS & LCS & MCS & $1.2 \% \mathrm{Si}$ & $9 \% \mathrm{Ni}$ & $13 \% \mathrm{Cr}$ \\
\hline room temperature & 19.9 & 19.8 & 12.2 & 9.07 & 7.96 & 7.24 \\
773 & 9.62 & 9.60 & 7.89 & 7.18 & 6.90 & 5.61 \\
973 & 5.53 & 5.70 & 5.03 & 4.81 & 4.65 & 3.93 \\
1176 & - & 5.84 & 5.89 & 4.94 & 5.79 & 5.74 \\
1273 & 6.14 & 6.21 & 5.99 & 5.38 & 6.08 & 5.84 \\
1375 & 6.44 & 6.37 & 6.18 & 5.69 & 6.11 & 5.72 \\
1572 & 6.60 & 6.84 & 6.46 & 5.56 & 6.25 & 6.00 \\
1622 & 6.59 & 6.81 & 6.19 & 5.35 & 6.20 & 6.06 \\
1676 & 6.52 & 6.88 & 6.02 & 5.69 & 5.95 & 5.71 \\
\hline
\end{tabular}

Table 5. Thermal diffusivity values (unit: $\mathrm{mm}^{2} \mathrm{~s}^{-1}$ ) of ultra low carbon steel (ULCS), low carbon (LCS), medium carbon steel (MCS), 1.2\% silicon steel $(1.2 \%$ $\mathrm{Si}), 9 \%$ nickel steel $(9 \% \mathrm{Ni})$ and $13 \%$ chromium steel $(13 \% \mathrm{Cr})$ in the temperature range of austenite phase fitted using a linear regression. The uncertainty of thermal diffusivity of six steels below $1375 \mathrm{~K}$ is $\pm 1.6 \%$. The uncertainty of thermal diffusivity of six steels above $1572 \mathrm{~K}$ is $\pm 5.8 \%$.

\begin{tabular}{|c|c|c|c|}
\hline \multirow{2}{*}{ Steel } & \multirow{2}{*}{$\begin{array}{c}\text { Temperature } \\
\text { range }(\mathrm{K})\end{array}$} & \multicolumn{2}{|c|}{ Regression $\alpha=\mathrm{b}_{0}+\mathrm{b}_{1} T$} \\
\cline { 3 - 4 } & $\mathrm{b}_{0}$ & $\mathrm{~b}_{1}$ \\
\hline ULCS & $1273 \leqq T \leqq 1622$ & 3.95 & $1.76 \times 10^{-3}$ \\
\hline $\mathrm{LCS}$ & $1176 \leqq T \leqq 1676$ & 3.20 & $2.30 \times 10^{-3}$ \\
\hline $\mathrm{MCS}$ & $1176 \leqq T \leqq 1676$ & 4.80 & $9.50 \times 10^{-4}$ \\
\hline $1.2 \% \mathrm{Si}$ & $1176 \leqq T \leqq 1676$ & 2.71 & $2.03 \times 10^{-3}$ \\
\hline $9 \% \mathrm{Ni}$ & $1176 \leqq T \leqq 1676$ & 4.80 & $9.28 \times 10^{-4}$ \\
\hline $13 \% \mathrm{Cr}$ & $1176 \leqq T \leqq 1676$ & 5.56 & $1.66 \times 10^{-4}$ \\
\hline
\end{tabular}

\section{Acknowledgements}

The authors wish to express their gratitude to Prof. $\mathrm{H}$. Ohtani, Dr. K. Yoshimi and Dr. T. Haraguchi, Center for Interdisciplinary Research Tohoku University, for their help on the thermal expansion measurements.

\section{Nomenclature}

$T_{\mathrm{r}}$ : Temperature rise of the rear surface of the specimen $(\mathrm{K})$

$T_{\mathrm{M}}$ : Maximum temperature rise of the rear surface of the specimen with no heat loss $(\mathrm{K})$

$Q: \quad$ Total energy absorbed by the specimen (J)

$C$ : Heat capacity of specimen $\left(\mathrm{J} \mathrm{K}^{-1}\right)$

$\sigma$ : Stefan-Boltzmann constant $\left(\mathrm{W} \mathrm{mm}^{-2} \mathrm{~K}^{-4}\right)$

$T_{0}$ : Steady-state temperature of specimen $(\mathrm{K})$

$l$ : Thickness of specimen at $T_{0}(\mathrm{~mm})$

$\rho:$ Density of the specimen $\left(\mathrm{g} \mathrm{mm}^{-3}\right)$

$C_{p}$ : Specific heat capacity of specimen $\left(\mathrm{Jg}^{-1} \mathrm{~K}^{-1}\right)$

$\varepsilon_{\mathrm{s}}: \quad$ Emissivity of the surface of specimen

$\alpha$ : Thermal diffusivity $\left(\mathrm{mm}^{2} \mathrm{~s}^{-1}\right)$

$\beta$ : Decrease in thickness of specimen for the measurement of thermal diffusivity $(\mathrm{mm})$

$l_{T}$ : Estimated thickness of specimen at elevated temperature of $T(\mathrm{~mm})$

$l_{\mathrm{RT}}$ : Thickness of specimen at room temperature for the measurement of thermal diffusivity (mm)

$L_{\mathrm{RT}}$ : Length of specimen at room temperature for the measurement of linear thermal expantion (mm)

$\Delta L: \quad$ Elongation of specimen at each measurement temperature $(\mathrm{mm})$ 


\section{$100 \Delta L / L_{\mathrm{RT}}:$ Percentage linear thermal expansion}

\section{REFERENCES}

1) Y. S. Touloukin, R. W. Powell, C. Y. Ho and M. C. Nicolaou: Thermophysical Properties of Matter., Vol. 10, IFI/PLENUM, New York, (1973), 82.

2) Y. S. Touloukin, R. W. Powell, C. Y. Ho and M. C. Nicolaou: Thermophysical Properties of Matter., Vol. 12, IFI/PLENUM, New York, (1973), 868.

3) W. J. Parker, R. J. Jenkins, C. P. Butler and G. L. Abbott: J. Appl. Phys., 32 (1961), 1679.

4) A. Cezairliyan, T. Baba and R. Taylor: Int. J. Thermophys., 15 (1994), 317.

5) Y. S. Touloukin, R. W. Powell, C. Y. Ho and M. C. Nicolaou: Thermophysical Properties of Matter., Vol. 12, IFI/PLENUM, New York, (1973), 157.
6) T. B. Massalski, H. Okamoto, P. R. Subramanian and L. Kacprzak: Binary Alloy Phase Diagrams, 2nd Ed., Vol. 1, ASM International, Materials Park, OH, (1990), 842.

7) T. B. Massalski, H. Okamoto, P. R. Subramanian and L. Kacprzak: Binary Alloy Phase Diagrams, 2nd Ed., Vol. 2, ASM International, Materials Park, OH,(1990), 1771.

8) M. D. Mathews, B. R. Ambekar and A. K. Tyagi: J. Nucl. Mater., 288 (2001), 83.

9) A. K. Tyagi, M. D. Mathews and R. Ramachandran: J. Nucl. Mater, 294 (2001), 198.

10) N. Yamada, R. Abe and M. Okaji: Meas. Sci. Technol., 12 (2001), 2121.

11) Handbook of Iron and Steel (Tekkobinran), ed. by ISIJ, Maruzen, Tokyo, (1981), 205.

12) Y. S. Touloukin, R. W. Powell, C. Y. Ho and M. C. Nicolaou: Thermophysical Properties of Matter., Vol. 10, IFI/PLENUM, New York, (1973), 337. 